\section{CARD10, a CEBPE target involved in granulocytic differentiation}

\author{
Pavithra Shyamsunder, ${ }^{1 *}$ Haresh Sankar, ${ }^{1}$ Anand Mayakonda, ${ }^{1}$ Lin Han, ${ }^{1,2}$ \\ Hazimah Binte Mohd Nordin, ${ }^{1}$ Teoh Weoi Woon, ${ }^{1}$ \\ Mahalakshmi Shanmugasundaram, ${ }^{1}$ Pushkar Dakle, ${ }^{1}$ Vikas Madan ${ }^{1 * \#}$ \\ and H. Phillip Koeffler $r^{1,3,4 \#}$
}

${ }^{1}$ Cancer Science Institute of Singapore, National University of Singapore, Singapore; ${ }^{2}$ Department of Medicine, Yong Loo Lin School of Medicine, National University of Singapore; ${ }^{3}$ Cedars-Sinai Medical Center, Division of Hematology/Oncology, UCLA School of Medicine, Los Angeles, CA, USA and ${ }^{4}$ Department of Hematology-Oncology, National University Cancer Institute of Singapore (NCIS), National University Hospital, Singapore

"VM and HPK share senior authorship

\section{ABSTRACT}

M aturation of granulocytes is dependent on controlled gene expression by myeloid lineage restricted transcription factors. CEBPE is one of the essential transcription factors required for granulocytic differentiation. Identification of downstream targets of CEBPE is vital to understand better its role in terminal granulopoiesis. In this study, we have identified Card10 as a novel target of CEBPE. We show that CEBPE binds to regulatory elements upstream of the murine Card10 locus, and expression of CARD10 is significantly reduced in Cebpe knock-out mice. Silencing Card10 in a human cell line and in murine primary cells impaired granulopoiesis, affecting expression of genes involved in myeloid cell development and function. Taken together, our data demonstrate for the first time that Card10 is expressed in granulocytes and is a direct target of CEBPE with functions extending to myeloid differentiation.

\section{Introduction}

Precise levels of progenitor cell proliferation versus lineage-committed differentiation is central to the balanced functioning of the hematopoietic system. ${ }^{1,2}$ Transcription factors are fundamental elements directing differentiation during hematopoietic development. ${ }^{3}$ The lineage and stage-restricted expression pattern of these factors underlines the need for precise regulation of their function. Major transcription factors regulating myeloid development include PU.1, GFI1, IRF8, RUNX1, SCL, TAL1 and the members of C/EBP family. Each of these lineage restricted factors are known to drive the expression of a panel of target genes. ${ }^{410}$

CEBPE is a member of the CCAAT/enhancer binding protein (C/EBP) family of transcription factors involved in hematopoietic cell development and induction of several inflammatory mediators. ${ }^{11,12}$ CEBPE is expressed in a stage-specific manner during myeloid differentiation and regulates transition from the promyelocyte to the myelocyte. ${ }^{13}$ This transcription factor is essential for secondary and tertiary granule formation in granulocytes. ${ }^{14}$ Germline mutations of the CEBPE gene have been detected in patients with neutrophil-specific granule deficiency. Their neutrophils display atypical bilobed nuclei, lack expression of granule proteins and these patients often have frequent bacterial infections. ${ }^{15,16}$ Cebpe knock-out mice resemble this clinical phenotype displaying a block in terminal differentiation and absence of secondary granule proteins. Cebpe KO mice develop normally, except that they fail to produce functional neutrophils and eosinophils. Neutrophils from these mice have impaired chemotaxis, bactericidal activity and mice typically die of infections between 3 and 5 months of age. ${ }^{9}$ The lack of secondary granule proteins in granulocytes from these mice impairs their responses to inflammatory signals characterized by an increase in circulating immature neutrophils and recurrent pyogenic infections. ${ }^{17}$

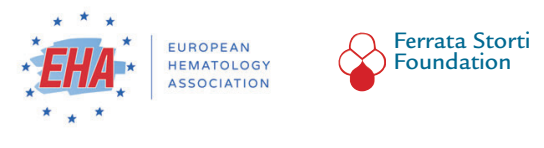

Haematologica 2018

Volume 103(8):1269-1277

\section{Correspondence:}

csips@nus.edu.sg or csivm@nus.edu.sg

Received: February 4, 2018.

Accepted: May 14, 2018.

Pre-published: May 17, 2018.

doi:10.3324/haematol.2018.190280

Check the online version for the most updated information on this article, online supplements, and information on authorship \& disclosures: www.haematologica.org/content/103/8/1269

\section{(C)2018 Ferrata Storti Foundation}

Material published in Haematologica is covered by copyright. All rights are reserved to the Ferrata Storti Foundation. Use of published material is allowed under the following terms and conditions:

https://creativecommons.org/licenses/by-nc/4.0/legalcode. Copies of published material are allowed for personal or internal use. Sharing published material for non-commercial purposes is subject to the following conditions:

https://creativecommons.org/licenses/by-nc/4.0/legalcode, sect. 3. Reproducing and sharing published material for commercial purposes is not allowed without permission in writing from the publisher. 
Identification of novel targets of CEBPE is imperative for a better understanding of granulocytic differentiation. In this study, we utilized ChIP-seq and RNA-seq data, the former to identify global DNA binding pattern of CEBPE and the latter to note expression changes of CEBPE deficient cells. Together, these techniques allowed identification of novel targets of CEBPE associated with myelopoesis.

One of the interesting targets of CEBPE that we identified in this analysis is Card10 (also called Carma3). The CARMA family has three members, CARMA1, CARMA2, and CARMA3. They contain a N-terminal CARD domain, followed by a coiled-coil domain (C-C), a PDZ domain, a SH3 domain, and a Guanylate Kinase-like (GUK) domain in the C-terminus. Although the CARMA proteins share a high degree of sequence similarity, they display a distinct tissue expression pattern. ${ }^{18}$ A study by Xin Lin et al., analysed microarray data of 353 human tissue samples and found that CARMA1 is primarily expressed in hematopoietic tissues such as spleen, thymus, and peripheral blood leukocytes. Expression of CARMA2 is specific to placenta, and CARMA3 (CARD10) is expressed in a wide range of tissues with modest expression detected in hematopoietic cells. ${ }^{19} \mathrm{CARD} 10$ has been described as a molecular link between G proteincoupled receptors and NF-кB. GPCR induced ubiquitination of IKK- $\gamma$ (NEMO) with concomitant activation of the IKK complex is completely defective in CARD10 deficient cells. ${ }^{18}$ Studies have demonstrated that CARD10 deficient murine embryonic fibroblasts have diminished lysophosphatidic acid (LPA) induced NF-KB activation and lowered IL-8 production. ${ }^{18}$ Similarly, inhibition of CARD10 in airway epithelial cells reduces LPA-mediated NF- $\mathrm{KB}$ activity and the secretion of NF- $\mathrm{KB}$ dependent cytokines, TSLP and CCL20, thus pointing to a role for CARD10 in initiating allergic inflammation..$^{20}$

In the present study, we show that Card10 is a direct target of CEBPE. We verify that CEBPE binds to a region upstream of Card10 gene and its expression is upregulated in the granulocytic population. We demonstrate that knock-down of Card10 in a human cell line and in murine progenitor cells causes a defect in granulocytic differentiation. Expression analysis of Card10 depleted murine progenitor cells revealed that knock-down of Card10 affected expression of key genes involved in granulopoiesis. Taken together, we identify Card10 as a novel target of CEBPE with a role in myeloid cell differentiation and function.

\section{Methods}

\section{Mice}

CEBPE knock out (KO) mice have been described previously. ${ }^{9}$ Mice were maintained on a C57BL/6J (B6) genetic background at the animal facility of Comparative Medicine Centre, National University of Singapore (NUS). CEBPE KO allele was genotyped using primers: GCTACAATCCCCTGCAGTCC, TGCCTTCTGCCCTTGTG and ATCGCCTTCTATCGCCTTCTTGACGAG. All mice experiments were approved by Institutional Animal Care and Use Committee, NUS, Singapore.

\section{Flow cytometry}

Single cell suspensions were incubated with fluorochrome-conjugated antibodies for $30 \mathrm{~min}$ on ice. Cells were washed with $2 \%$ FBS/PBS and resuspended in SYTOX Blue Dead Cell Stain
(ThermoFisher Scientific). Flow cytometric analysis was performed on FACS LSR II flow cytometer (BD Biosciences), and sorting of cells was performed on FACS Aria cell sorter (BD Biosciences). Data were analyzed using FACSDIVA software (BD Biosciences).

\section{ChIP-PCR}

DNA-protein complexes were cross-linked with $1 \%$ formaldehyde at room temperature for $10 \mathrm{~min}$, followed by quenching with $0.2 \mathrm{M}$ glycine for $5 \mathrm{~min}$. Cells were lysed and chromatin was sonicated in Lysis buffer ( $1 \% \mathrm{SDS}, 50 \mathrm{mM}$ Tris-HCl, $5 \mathrm{mM}$ EDTA) at $4^{\circ} \mathrm{C}$ using Diagenode Bioruptor. Sheared-sonicated chromatin was incubated overnight at $4^{\circ} \mathrm{C}$ with antibodies against Cebpe and a mixture of Dynabeads Protein A and Protein G (1:1). Bead-chromatin complexes were washed, and the chromatin was eluted in $1 \%$ SDS, $0.1 \mathrm{M}$ sodium bicarbonate and reverse-crosslinked at $65^{\circ} \mathrm{C}$ for 16 hours. Immunoprecipitated DNA was extracted using OIAquick PCR Purification Kit (Oiagen) and quantified using Qubit Fluorometer (Life Technologies). Input and immunoprecipitated DNA were amplified using three different primer pairs. Primer sequences used for ChIP-PCR can be found in Online Supplementary Table S4.

\section{Electrophoretic mobility shift assay}

293T cells were transfected with either $1 \mu \mathrm{g}$ pcDNA3.1(-) empty vector or Cebpe expressing vector in $100 \mathrm{~mm}$ dishes using Jetprime transfection reagent (Polypus) according to manufacturer's instructions. After transfection, cells were cultured for 48 hours, and nuclear extracts were prepared using NE-PER reagent (Thermo Scientific). Double-stranded oligonucleotide probes were labelled using 3' Biotin End labelling kit (Thermo Scientific) following the manufacturer's instructions. Following biotinylated probes were used: CEBP consensus (5'-GATCCATATCCCTGATTGCGCAATAGGCTCAAAA); Card10 (5'- GAATGAGCCGATTGCTGCAACCTGGAAGG); Mutant Card10 (5'- GAATGAGCCGGCCTTGGGGCCCTGGAAGG) along with 100 fold molar excess of corresponding cold competitor. EMSA was carried out using LightShift Chemiluminescent EMSA Kit (Thermo Scientific). DNA-protein complexes were resolved on native $10 \%$ polyacrylamide-TBE gels.

\section{Luciferase reporter assay}

A 500 bp fragment upstream of Card10 gene (encompassing the CEBPE binding site identified in ChIP-seq) was amplified from genomic DNA extracted from murine bone marrow cells and subcloned into pGL4-Basic vector (Promega, Madison, WI). NIH/3T3 cells were transfected with pCDNA-Cebpe along with either pGL4 basic vector or pGL4-Card10 vector (-7kb peak) using Lipofectamine Plus (Life Technologies). Renilla basic vector was co-transfected as a control for normalization of luciferase activity; luciferase was measured 24 hours after transfection using Promega Dual-Glo assay kit, as per the manufacturer's instructions.

\section{Isolation, culture and differentiation of Lin-Kit bone marrow cells}

Murine Lin-Kit ${ }^{+}$cells were isolated from the bone marrow of C57BL/6 mice. Briefly, bone marrow cells were flushed from femurs and tibias using PBS containing 5\%FBS; red cells were lysed and anti-rat IgG Dynabeads (Invitrogen) were used to deplete lineage +ve cells. Lin-Kit cells were sorted on FACS Aria (BD Biosciences). Sorted cells were cultured in the presence of IL3, IL6 and SCF. To induce granulocytic differentiation, $10 \mathrm{ng} / \mathrm{mL}$ GM-CSF was added to the culture medium. Granulocytic differentiation was monitored using flow cytometry at days 3, 5 and 7 of GM-CSF treatment. 


\section{Short hairpin RNA (shRNA) interference}

To obtain Card10 knock-down in NB4 cells and murine progenitor cells, human and murine Card10 shRNA were cloned in the pLKO.1 lentiviral vector. Briefly, for virus production, 293T cells were seeded in a $100 \mathrm{~mm}$ dish, one day before transfection in DMEM medium supplemented with 10\% FBS. Plasmid DNA (either non-target shRNA or Card10 shRNA) was transfected along with pCMV-dr8.2 and pMD2.G using Lipofectamine 2000 transfection reagent (Invitrogen, Carlsbad, CA, USA). After 4 hours, the transfection medium was replaced with DMEM medium supplemented with $10 \%$ FBS. Cell culture supernatants containing the lentivirus were collected at $48 \mathrm{~h}$ and $72 \mathrm{~h}$ post-transfection. Cells were transduced with lentiviral particles in the presence of $8 \mu \mathrm{g} / \mathrm{ml}$ polybrene (Sigma-Aldrich) for $24 \mathrm{~h}$. Transduced cells were selected with puromycin $(1 \mu \mathrm{g} / \mathrm{ml}$ for NB4 and $2 \mu \mathrm{g} / \mathrm{ml}$ for mouse $\mathrm{Lin}^{-} \mathrm{Kit}^{+} \mathrm{BM}$ cells) for a week.

\section{RNA isolation, cDNA conversion and QPCR}

RNA from sorted murine granulocytes, progenitor populations or NB4 cells was isolated using either RNeasy Micro or Mini Kit (Qiagen) depending on the number of cells. cDNA was prepared using MuLV Reverse Transcriptase (Thermo Fisher Scientific). Primer sequences used for quantitative RT-PCR are listed in Online Supplementary Table S1.

\section{RNA sequencing and expression analysis}

cDNA libraries were prepared from poly-A selected RNA using Truseq RNA sample kit (Illumina). Libraries were sequenced on HiSeq 4000 and 100 bp paired-end reads were aligned to murine reference transcriptome (GRCm38/mm10; Ensemble version 84) using Kallisto (version 0.43.0). Transcript level fragment counts were summarized at the gene level using TxImport Bioconductor package, and differential analysis was performed using DESeq2. Gene expression was quantified in FPKM units using DESeq2 FPKM command and was used for all downstream analysis and plotting. All other test-statistics and plotting were performed using R 3.4.0. Gene Ontology (GO) was performed on differentially expressed genes using goseq Bioconductor package (version 1.20.0), which accounts for bias due to gene length. Resulting $P$-values were adjusted for False Discovery Rate (FDR). For GSEA analysis, we used all "active transcripts" with mean expression of 0.5 FPKM to identify significantly enriched gene sets among MSigDB C2 gene sets.

\section{Statistical analysis}

Two-sided, unpaired Student $t$-test was used to determine the statistical significance of experimental results. Data represented as mean \pm SD. $P$ values $<0.05$ are considered statistically significant.

A

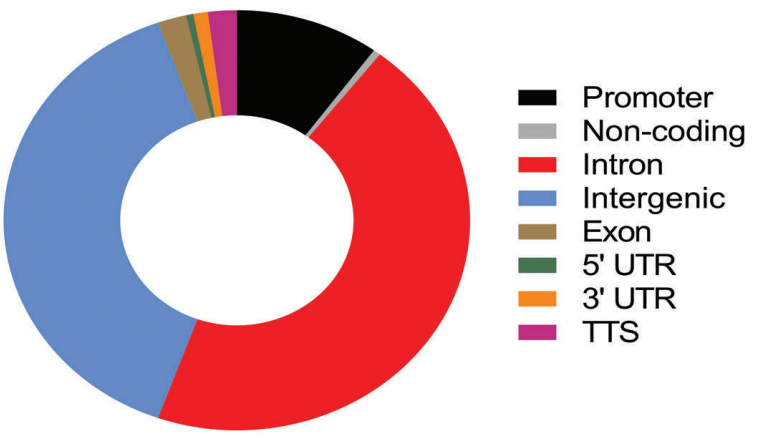

B

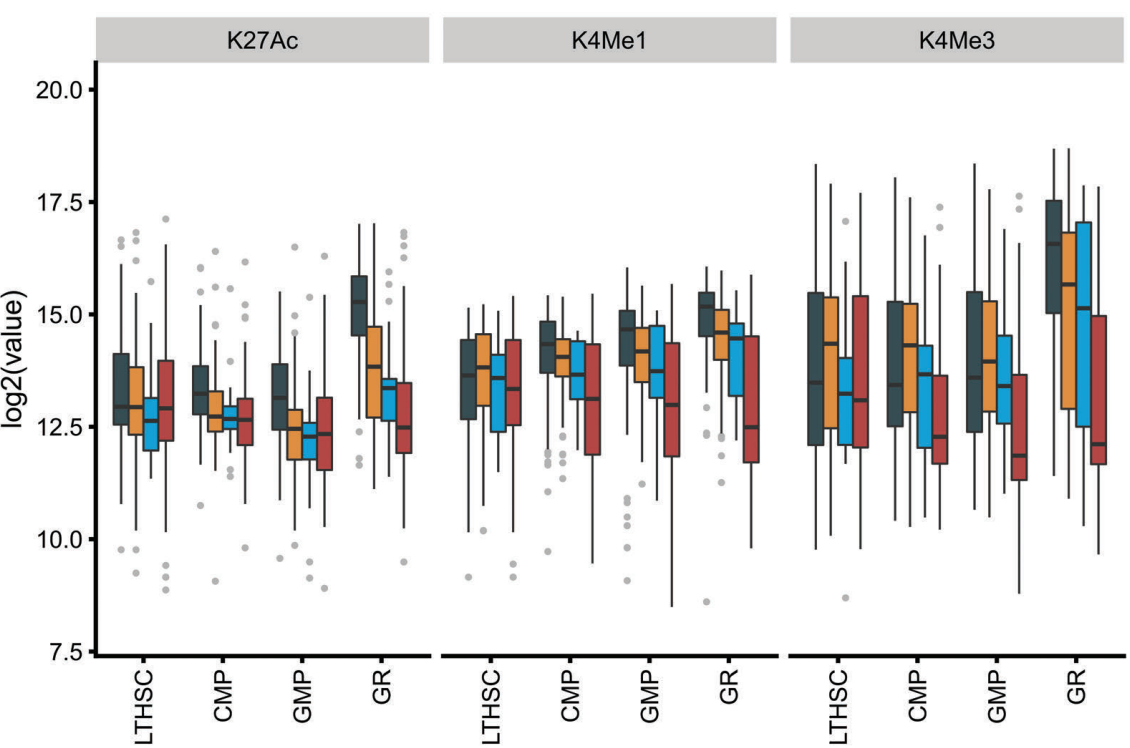

Figure 1. Characterization of CEBPE ChIP-Seq peaks. (A) Genomic annotation of the 40,517 CEBPE binding sites to the murine genome according to known RefSeq genes. (B) Box plots depict ChIP-seq signal intensities for histone marks in multiple hematopoietic cells ${ }^{22}$ for the 312 genes downregulated in Cebpe KO immature granulocytes. Histone marks were meas ured +/- $1 \mathrm{~kb}$ around CEBPE peaks. Peaks were classified based on the location of CEBPE binding. (i) Promoter peak ( $+1 \mathrm{~kb}$ to $-100 \mathrm{bp}$ ) (ii) Intronic peak (iii) Intergenic peak within $10 \mathrm{~kb}$ of the TSS (iv) Intergenic peak beyond $10 \mathrm{~kb}$ of the TSS.

Promoter 白 Intron 追 Intergenic<10kb fromTSS 白 Intergenic $>10 \mathrm{~kb}$ fromTSS 


\section{Results}

CEBPE predominantly binds at intergenic and intronic regions and regulates gene expression

We previously identified genome-wide CEBPE binding sites in unfractionated mouse bone marrow cells using chromatin immunoprecipitation followed by sequencing (ChIP-seq). ${ }^{21}$ Total bone marrow cells are composed of approximately $40-50 \%$ of mature granulocytes that express high levels of CEBPE. Further analysis of ChIP- seq data revealed that the overall binding pattern of CEBPE was similar to other CEBP factors, with increased binding
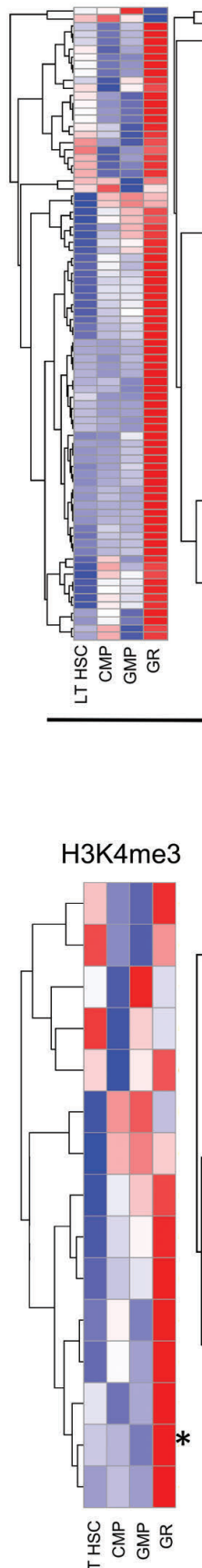

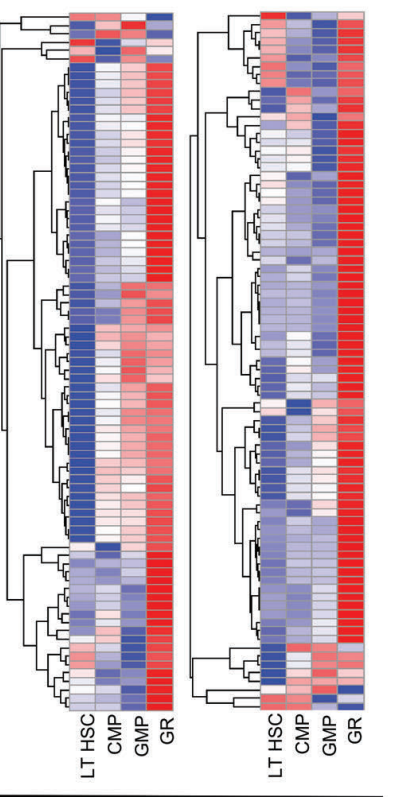

Promoter
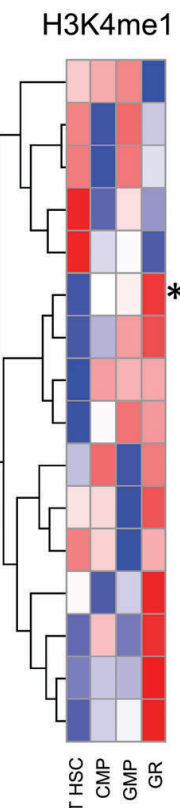

H3K27ac

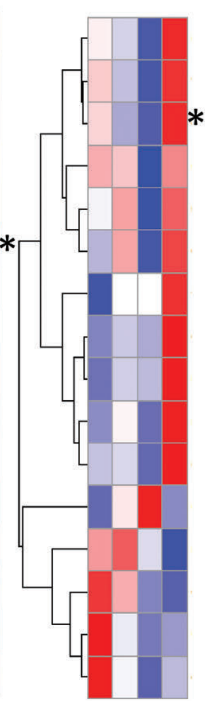

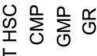

Intergenic $<10 \mathrm{~kb}$ from TSS

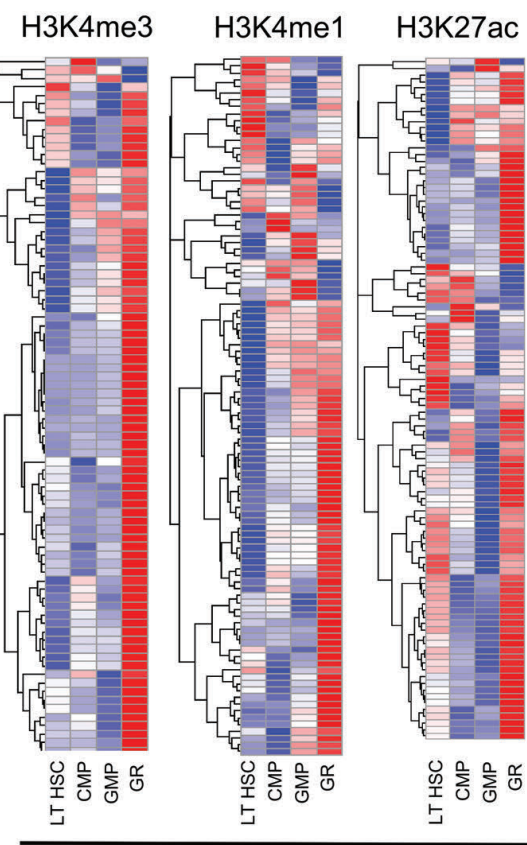

Intronic

\section{H3K4me3}

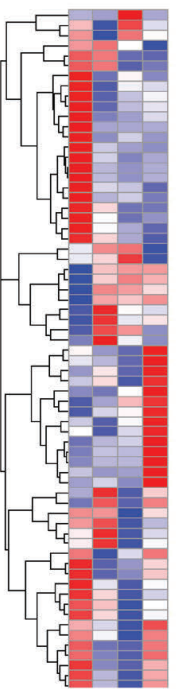

H3K4me1

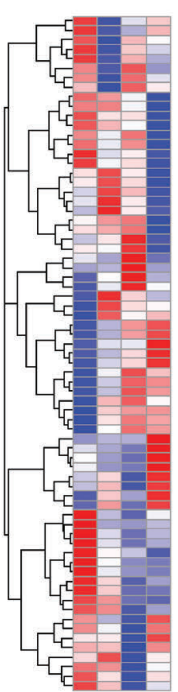

H3K27ac

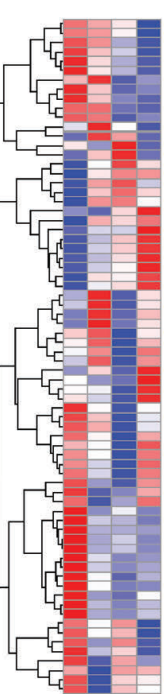

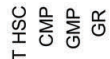

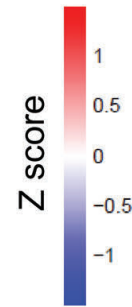

Figure 2. Genes bound by CEBPE have an activation signature in granulocytes. Heat maps of ChIP-Seq signal intensities of histone marks in long term hematopoetic stem cells (LT-HSC), common myeloid progenitors (CMP), granulocyte-monocyte progenitor (GMP) and granulocytes (Gr) populations for genes whose expression is downregulated in CEBPE KO cells and categorised according to location of binding of CEBPE to the promoter, intron, intergenic region <10kb and > $10 \mathrm{~kb}$ of the nearest gene TSS. ${ }^{22}$ *in the figure represents Card10. 
at intergenic (37\%) and intronic regions (46\%) and only $10 \%$ of the sites located in core promoter regions (Figure 1A). We integrated the gene expression profile of granulocytes from Cebpe WT and KO mice (RNA-seq) and CEBPE ChIP-seq analysis. A total of 312 genes were downregulated in the absence of CEBPE. Of these, 93 genes had a CEBPE promoter peak, 103 genes had an intronic peak and 116 genes had an intergenic peak. Of the 116 peaks, 18 genes had a peak within $10 \mathrm{~kb}$ from the transcriptional start site (TSS), while 98 genes had a peak beyond $10 \mathrm{~kb}$ from the TSS (Online Supplementary Table S1).

As genome-wide distribution of enhancer and promoter associated histone modifications provides a reliable signature of cell identity, we further analysed the 312 genes for histone marks in the hematopoietic lineage using a previously published data set. ${ }^{22}$ We used ChIP-seq data for three histone marks; H3K4me3 (mark of active promoters), H3K4me1 (mark of poised enhancers) and H3K27ac (mark of poised and active enhancers) and analysed the distribution of these marks for the 312 genes in long term hematopoetic stem cells (LT-HSC), common myeloid progenitors (CMP), granulocyte-monocyte progenitor (GMP) and granulocytes (Gr). Expectedly, comparison of
H3K27ac, H3K4me1 and H3K4me3 profiles showed that the majority of genes with promoter binding of CEBPE had a transcriptional activation signature in the granulocytes but not in (LT-HSC), myeloid precursors (CMP and GMP populations) (Figure 1B). Cumulative assessment of $Z$ scores revealed that in granulocytes, an overall increased signal intensity for all three active histone marks was detected in genes with either intronic or intergenic occupancy within $10 \mathrm{~kb}$ of the TSS, as compared with genes with peaks beyond 10kb of the TSS (Online Supplementary Figure S1).

This analysis further emphasizes the role of CEBPE as a transcription factor essential for granulopoiesis and identifies a set of genes that might be regulated by CEBPE, with ChIP-seq peaks at non-promoter regions.

\section{Card10/Carma3 is regulated by CEBPE in the granulocytic population}

Among the 18 genes that had an intergenic binding of CEBPE within $10 \mathrm{~kb}$ of the TSS, Card10 was an interesting target which harbors active histone marks and is exclusively expressed in granulocytes and monocytes (Online Supplementary Figure S2A).
A

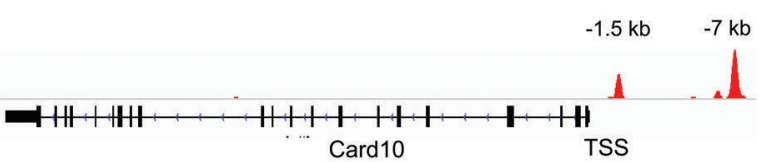

C

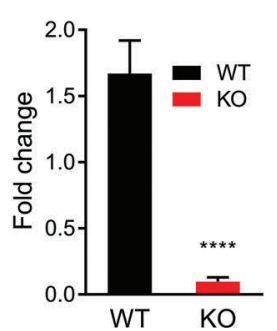

D

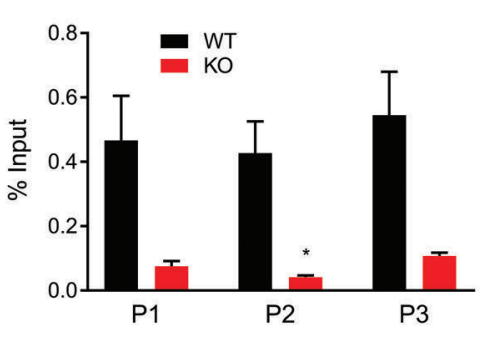

B

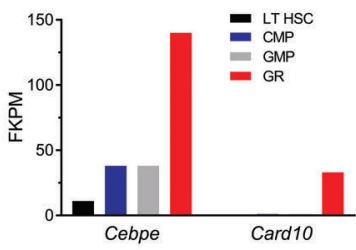

E

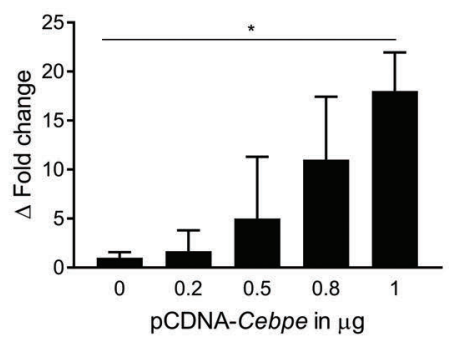

Card10 oligo Mutant oligo Cebpe oligo

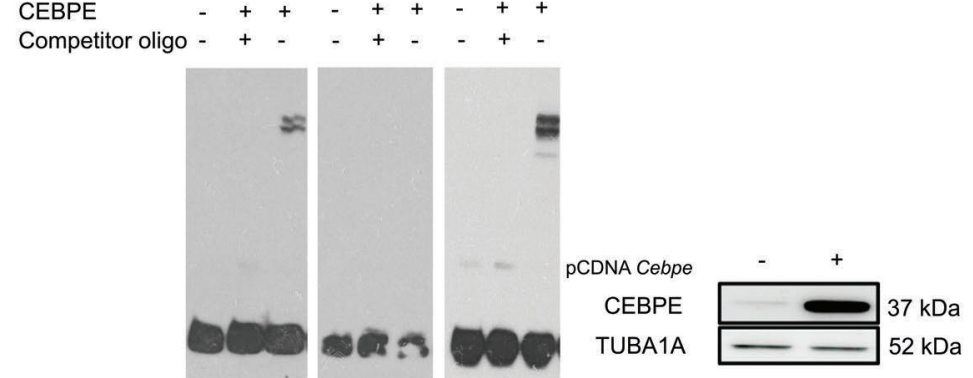

Figure 3. Card10 is a direct target of CEBPE. (A) Illustration of CEBPE binding peaks at the mouse Card10 locus. Binding sites located around -1.5kb and $-7 \mathrm{~kb}$ of the Card10 transcription start site are depicted in red. (B) FPKM values (RNA-seq) of Cebpe and Card10 in LT-HSC, CMP, GMP and Granulocytes (Gr) (https://www.ebi.ac.uk/gxa/experiments/E-MTAB-3079). (C) RT-PCR validation of transcript levels of Card10 in sorted immature granulocytes from wildtype and Cebpe KO mice. (D) ChIP-PCR validates binding of CEBPE to the $-7 \mathrm{~kb}$ region of Card10 gene using three primer pairs. Data are presented as percentage of input. (E) pGL4 basic luciferase vector containing a 500bp fragment harboring the -7kb CEBPE binding region was co-transfected with different amounts (0.2, 0.5, 0.8, and $1 \mu \mathrm{g}$ ) of pcDNA-Cebpe into NIH/3T3 cells. Luciferase activities were assayed 24 hours after transfection. Results represent fold induction of relative luciferase activity after normalization to Renilla control of two independent experiments, each done in triplicate. (F) Left panel; EMSA was performed with wild-type CEBPE binding sequence (CARD10 oligo), mutant CEBPE binding sequences (mutant oligo) and consensus CEBP binding sequences (CEBP oligo). Biotin-labelled probe was mixed with protein extracts from $293 \mathrm{~T}$ cells transfected with either an empty vector or an expression vector for CEBPE, and the reaction mixtures were resolved on native $10 \%$ polyacrylamide-TBE gel. Cold competition was carried out with 100-fold molar excess of unlabelled oligo. Right panel; Western blot of $293 T$ cell lysate $+/$ - transfection with CEBPE expression vector used for EMSA assay. Alpha tubulin (TUBA1A) was used as loading control. ****P<0.0001, $* P<0.05$ 


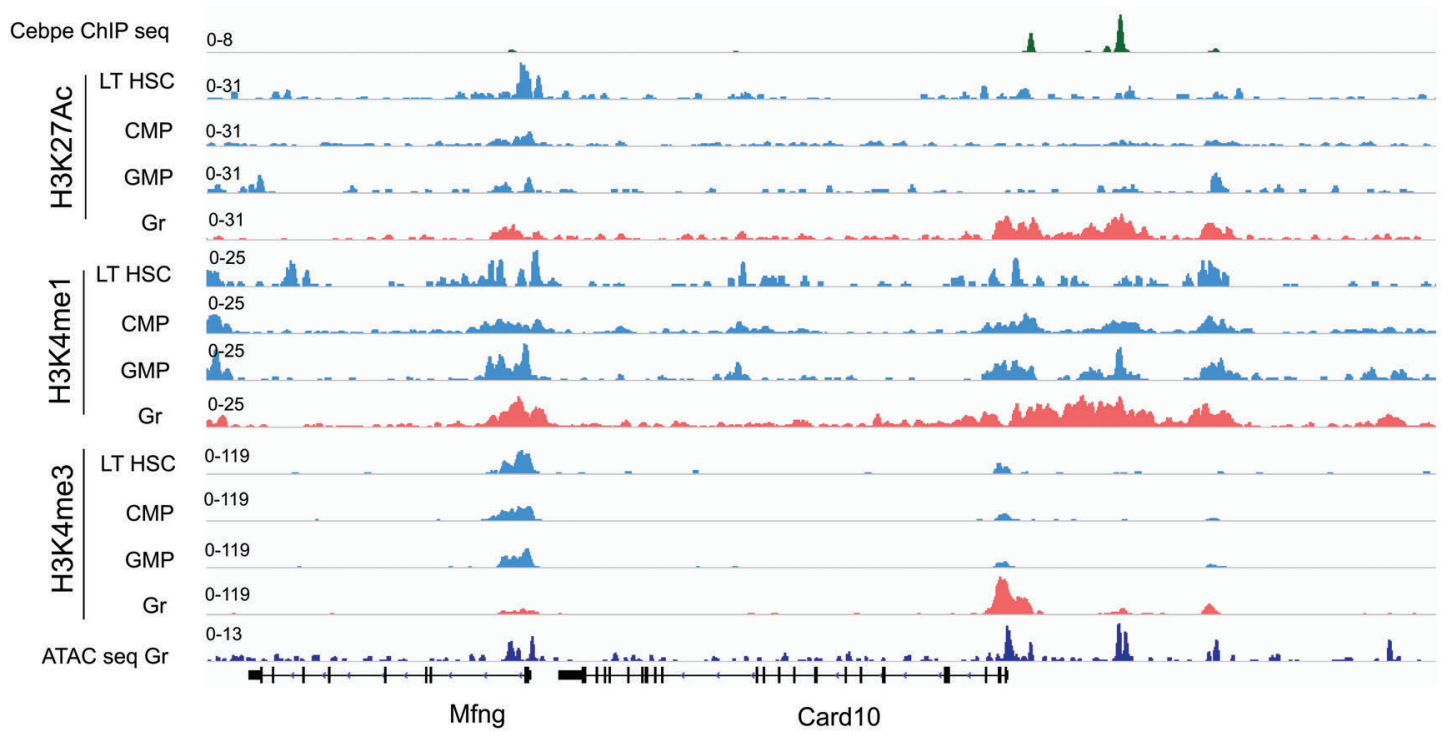

Figure 4. Card10 locus exhibits transcriptional activation signature in granulocytes. H3K27ac, H3K4me1 and H3K4me3 ChIP-seq signals in LT-HSC, CMP, GMP and granulocytes (Gr) and IGV track of ATAC-seq profile of sorted granulocytes (bottom track). Tracks are represented in frame with the CEBPE ChIP-seq peak of Card10 gene. ${ }^{22}$

We identified that CEBPE binds upstream of Card10 gene (-1.5 kb and $-7 \mathrm{~kb})$ (Figure $3 \mathrm{~A})$. Interestingly, expression profile of Card10 and Cebpe followed a similar pattern, with the highest level of expression observed in granulocytes (https://www.ebi.ac.uk/gxalexperiments/E-MTAB3079) (Figure 3B). Expression of Card10 was significantly lower in immature granulocytes from Cebpe $\mathrm{KO}$ mice compared with the same stage of differentiation of the wild type mice (Figure 3C, Online Supplementary Figure $S 2 B)$.

On scanning the ChIP-seq data, peaks occurred at the $7 \mathrm{~kb}$ and $-1.5 \mathrm{~kb}$ regions upstream of Card 10 gene. We also verified the binding of Cebpe to know targets such as Lactoferrin (Ltf) and Neutrophilic granule precursor protein (Ngp) (Online Supplementary Figure S3A). Examination of the nucleotide sequence using ConSite (an online software tool to predict transcription factor motifs) revealed two CEBPE motifs within the $-7 \mathrm{~kb}$ peak and one CEBPE motif was detected in the $-1.5 \mathrm{~kb}$ peak (Online Supplementary Figure S3B). The $-7 \mathrm{~kb}$ region had stronger binding of CEBPE compared with the $-1.5 \mathrm{~Kb}$ region; and therefore, the region was further studied. CEBPE binding to the $-7 \mathrm{~kb}$ peak was validated using ChIP-PCR in bone marrow cells from Cebpe WT mice; and this binding was significantly reduced in the $\mathrm{KO}$ cells (Figure $3 \mathrm{D}$ and Online Supplementary Figure S4).

To assess whether the interactions of CEBPE is functionally relevant, luciferase reporter assay and electrophoretic mobility shift assay (EMSA) were performed. Luciferase reporter assay with a 500 bp fragment containing the CEBPE motif sequence transfected into NIH/3T3 cells revealed that CEBPE was able to transactivate the luciferase reporter in a dose-dependent manner (Figure 3E). Next, biotinylated oligos encompassing the putative CEBP motif were designed and EMSA assays were performed. Incubation of biotinylated oligos with nuclear extract from cells ectopically expressing CEBPE caused a shift in migration of the biotinylated oligos. This shift dis- appeared when the nuclear extract complex was incubated with either 100-fold molar excess of unlabelled competitor oligos (cold competition) or with biotinylated oligos harboring a mutation in CEBP motif (Figure 3F). Taken together, these results indicate that Card10 is a direct target of CEBPE.

\section{Epigenetic landscape and expression of Card10 reveals an exclusive signature in granulocytes}

Comparison of H3K27ac, H3K4me1 and H3K4me3 occupancy at Card 10 locus (at the core promoter and $-7 \mathrm{~kb}$ CEBPE binding region) in LT-HSC, CMP, GMP and granulocytes, revealed transcriptional activation signatures exclusively in granulocytes (Figure 4). ATAC-Seq of sorted granulocytes ${ }^{22}$ also indicated that the region bound by CEBPE is located in open chromatin (Figure 4). These findings along with exclusive expression of Card10 in granulocytes suggests a role in myeloid differentiation.

\section{CARD10 regulates neutrophil differentiation in vitro}

Since Card 10 expression is controlled by CEBPE, we hypothesized that CARD10 may have a role in granulopoesis. To test this hypothesis, CARD10 expression was stably silenced using short hairpin RNA (shRNA) in NB4 cells, an acute promyelocytic leukemia cell line that can be differentiated into granulocytes in the presence of all-trans retinoic acid (ATRA) (Figure 5A). We observed that knockdown of CARD10 resulted in a reduced proportion of CD11 $b^{+}$cells upon induction with ATRA (control shRNA: 93\% and CARD10 KD: 83\% (sh4) and 63\% (sh5) 4 days incubation). Although the data are not statistically significant, we did observe a trend towards impaired differentiation following Card10 knock-down (Fig. 5B). In parallel as a control, we also verified granulocytic differentiation after ATRA induction of CEBPE knock-down cells (Online Supplementary Figure S5A and S5B). Viability assay with control and Card10 KD cells revevaled no major effect on cell proliferation (Online Supplementary Figure S5C). 


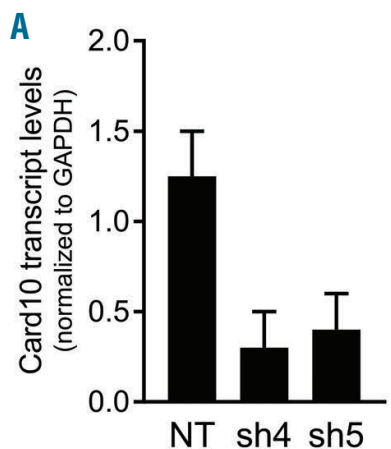

C

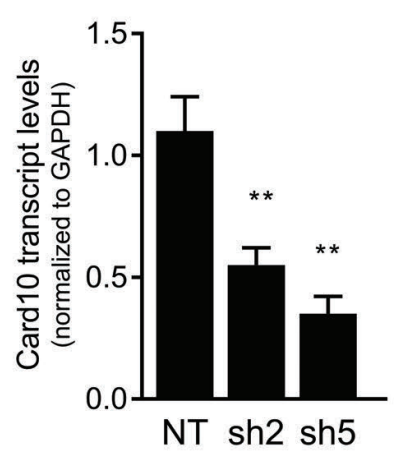

B

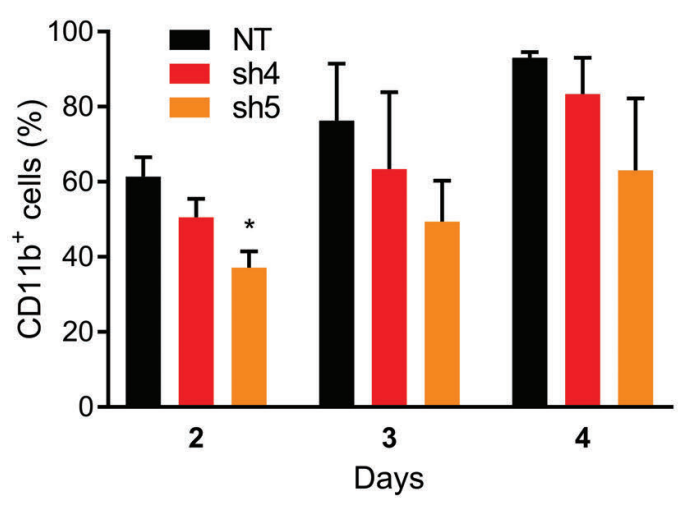

D

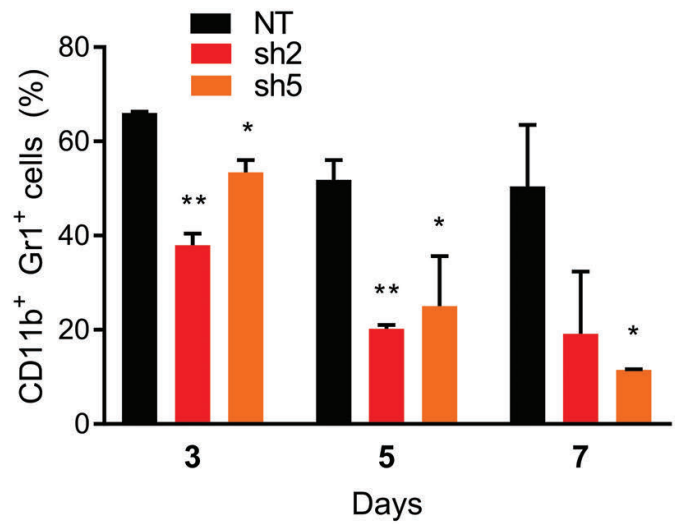

Figure 5. Knock down of CARD10 impairs myeloid differentiation. (A) RT-PCR analysis of CARD10 expression in human NB4 cells transduced with either non-target shRNA (NT) or CARD10 specific shRNAs (sh4 and sh5). Y-axis represents relative expression of CARD10 normalized to transcript levels of GAPDH (B) Proportion of $\mathrm{CD} 11 \mathrm{~b}^{+}$NB4 cells stably transduced with either control (NT) or CARD10 shRNA and cultured with $1 \mu \mathrm{M}$ ATRA for different duration. (C) RT-PCR validation of Card10 knock-down in murine Lin-Kitt bone marrow (BM) cells. Y-axis represents relative expression of Card10 normalized to Gapdh. (D) Granulocytic differentiation of Lin-Kit' $\mathrm{BM}$ cells stably expressing either NT or Card10 shRNAs (sh2 or sh5) in response to $10 \mathrm{ng} / \mathrm{mL}$ of $\mathrm{GM}-\mathrm{CSF}$. Differentiation was monitored by flow cytometric quantification of $\mathrm{CD} 11 \mathrm{~b}^{+} \mathrm{Gr} 1^{+}$cells. Results are average of three independent experiments. $* P<0.05, * * P<0.01$.

\section{Knock-down of CARD10 impairs granulocytic} differentiation of murine Lin-Kit' ${ }^{+}$BM cells

We evaluated further the role of CARD10 in granulocytic differentiation. shRNAs targeting murine Card10 were initially screened in NIH/3T3 cells (Online Supplementary Figure S6A) and two shRNAs that silenced Card10 were used to transduce murine Lin-Kit bone marrow (BM) cells. These shRNA sequences also robustly reduced the expression of Card10 in murine Lin-Kit ${ }^{+}$BM cells (Figure 5C). Lin-Kit ${ }^{+}$myeloid progenitors were transduced with either control or mouse Card10 shRNAs and grown in the presence of IL3, IL6, SCF and GM-CSF. The proportion of $\mathrm{CD}_{11} \mathrm{~b}^{+} \mathrm{Gr}^{+}$cells were analysed at different time points using flow cytometry. Our analysis revealed that Card10 knock-down resulted in significantly lower proportion of granulocytes at all the time points tested (control shRNA: $50 \%$, Card $10 \mathrm{KD}: 19 \%$ (sh2) and 11\% (sh5) at 7 days) (Figure 5D and Online Supplementary Figure S6B). This further illustrated a role for CARD10 in myeloid cell differentiation.

\section{Knock-down of Card10 affects expression of myeloid-specific genes}

To understand the changes in gene expression following Card10 knock-down, RNA-Seq of bulk control and Card10 knock-down Lin-Kit ${ }^{+}$BM cells was performed. Ninetyfour genes were downregulated and 68 genes were upregulated following Card10 knock-down $(\mathrm{FDR}<0.1)$ (Figure 6A). Gene Ontology analysis of differentially expressed genes revealed enrichment of genes involved in immune response, inflammatory response, leukocyte migration and response to external stimuli (Figure $6 \mathrm{~B}$ and Online
Supplementary Table S2). Furthermore, GSEA analysis of downregulated genes revealed a strong enrichment for genes involved in myeloid development (Figure 6C). Expression signature of Card10 knock-down Lin-Kit ${ }^{+}$BM cells (carried out with two independent shRNAs) was compared to that of Cebpe $\mathrm{KO}$ immature granulocytes. Twenty-nine of the 94 genes downregulated in Card10 knock-down cells were also downregulated in Cebpe KO cells (Figure 6D). We validated the lower expression of prominent myeloid specific genes using RT-PCR in Card10 $\mathrm{KD}$ Lin-Kit ${ }^{+} \mathrm{BM}$ cells (Figure $\left.6 \mathrm{E}\right)$. These results show that loss of CARD10 affects expression of genes implicated in myelopoesis.

\section{Discussion}

CEBPE is a transcription factor essential for functional maturation of granulocytes. Patients with neutrophil-specific granule deficiency have mutations of the CEBPE gene. ${ }^{15,16}$ Cebpe knockout mice recapitulate the disease and fail to produce terminally differentiated granulocytes. Previous studies from our group and others have identified several key targets of CEBPE, ${ }^{23-26}$ including a comprehensive approach that curated a list of CEBPE targets by comparing CEBPE binding sites to the gene expression changes in sorted granulocytes from Cebpe WT and KO cells. ${ }^{21}$ The present study extends the analysis to identify additional novel targets of CEBPE including genes with intronic or intergenic binding. Analysis of histone modifications at loci of genes with intronic/intergenic peaks showed that a majority of them had an active epigenetic signatures in the granulocytic population, suggesting these 
A

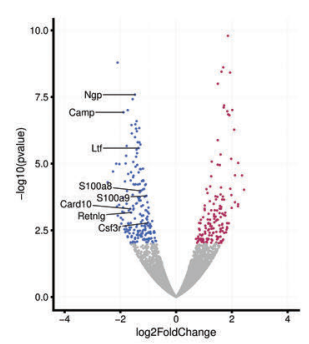

D

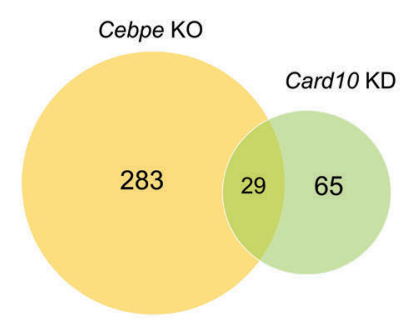

B

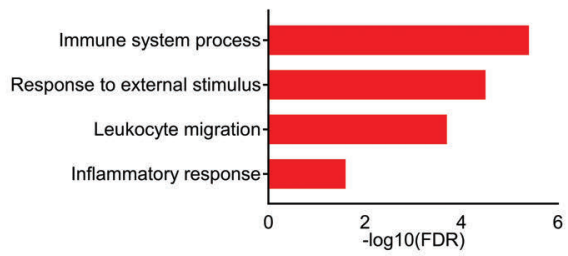

C

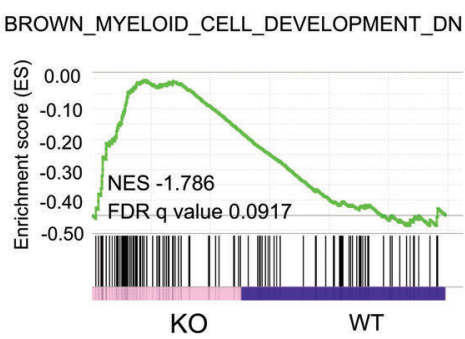

E

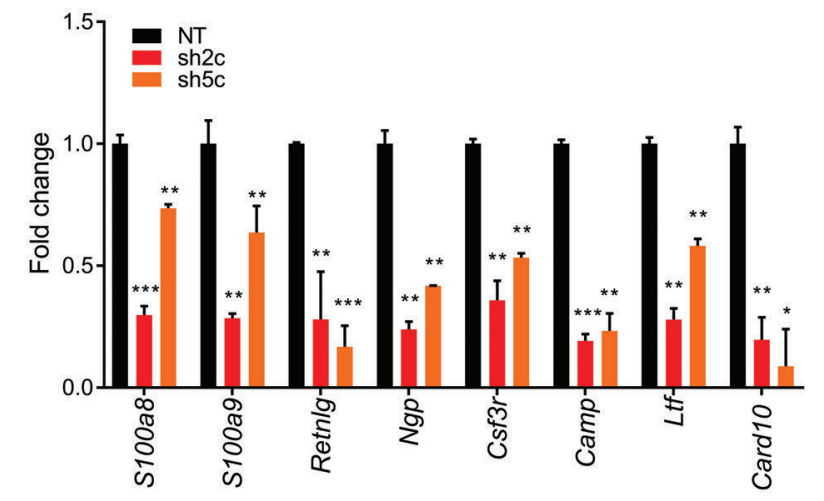

Figure 6. Gene expression changes in Card10 deficient murine Lin-Kit ${ }^{+}$bone marrow cells. (A) Volcano plot depicts genes differentially expressed in Card10 knockdown Lin ${ }^{-}$Kit $^{+}$BM cells compared to the WT cells (RNA-Seq). (B and C) Gene Ontology analysis (B) and GSEA plot (C) reveals enrichment of genes involved in myeloid development. (D) Venn diagram shows overlap of downregulated genes in WT vs. Cebpe KO (sorted immature granulocytes) compared to Card10 KD Lin-Kit ${ }^{+}$BM cells. (E) RT-PCR measuring the transcript levels of myeloid specific genes in NT and Card10 knock-down Lin-Kit ${ }^{+}$BM cells. Y-axis represents relative expression of genes normalized to Gapdh. ${ }^{*} P<0.05, * * P<0.01, * * * P<0.001$.

genes are also positively regulated by CEBPE during differentiation of granulocytes. Amongst these, Card10 was investigated as a putative novel target of CEBPE.

We identified binding of CEBPE at two sites upstream of the Card10 gene and hypothesized that CEBPE might regulate transcription of Card10. We verified occupancy of CEBPE to the $-7 \mathrm{~kb}$ region upstream of Card10 gene using ChIP-PCR, luciferase reporter assay and EMSA. We observed impaired granulocytic diffferentiation following Card10 knock-down in both human (NB4) and murine $\left(\mathrm{Lin}^{-} \mathrm{Kit}^{+}\right) \mathrm{BM}$ cells. These findings suggest that CARD10 may be an important mediator of granulocytic differentiation.

To understand how deficiency of CARD10 causes perturbation in myeloid differentiation, we performed expression analysis of Card10 knock-down and murine Lin-Kit ${ }^{+}$bone marrow cells. RNA-seq data showed that knock-down of Card10 affected expression of genes involved in myeloid development and function. Among the genes downregulated in Card10 knock-down cells, 29 were also downregulated in Cebpe $\mathrm{KO}$ granulocytes and analysis of their gene expression pattern revealed that these genes were exclusively expressed in the granulocytic population (Online Supplementary Table S3).

Migration of neutrophils to an inflammatory site is modulated by the activation of NFKB signalling. ${ }^{27,28}$ Published literature has documented a role for CARD10 as a scaffold protein for the NFKB signalling pathway, with no reference of its expression or role in granulopoiesis. ${ }^{19}$ The present study highlights that Card10 expression is regulated by CEBPE and that Card10 deficient cells have impaired granulopoiesis accompanied by reduced transcript levels of genes important in myeloid cell function. The impaired migration and phagocytosis observed in Cebpe KO mice may be contributed by lowered levels of Card10.

We have previously shown that stimulating activity of CEBPE in vivo can enhance the antimicrobial function of the body. ${ }^{29}$ As microbes evolve to discover new ways to become resistant to antibiotics, enhancing innate immunity becomes more important. Whether the transcriptional changes observed upon Card10 knock-down is an effect of loss of mature granulocytes or a direct effect of Card10 function, warrants further analysis. In summary, this study demonstrates that Card10 is a novel CEBPE target gene and is implicated in granulocytic differentiation.

\section{Acknowledgments}

We would like to thank the staff of Comparative Medicine, NUS for their support in maintenance of mouse colonies and experiments involving mice. We would also like to acknowledge the expert help and support from the FACS facility at CSI, Singapore. We thank the Melamed Family and Reuben Yeroushalmi for their generous support.

\section{Funding}

This work was also funded by the Leukemia Lymphoma Society of America, (the Singapore Ministry of Health's National Medical Research Council (NMRC) under its Singapore Translational Research (STaR) Investigator Award 
to H. Phillip Koeffler (NMRC/STaR/0021/2014), Singapore Ministry of Education Academic Research Fund Tier 2 (MOE2013-T2-2-150), the NMRC Centre Grant awarded to National University Cancer Institute of Singapore (NMRC/CG/012/2013) and the National Research Foundation Singapore and the Singapore Ministry of Education under its Research Centres of Excellence initiatives. This research is also supported by the RNA Biology Centre at the Cancer Science Institute of Singapore, NUS, as part of funding under the Singapore Ministry of Education's Tier 3 grants, grant number MOE2014-T3-1-006. This paper is dedicated to the memory of Parker Hughes.

\section{References}

1. Orkin SH, Zon LI. Hematopoiesis: an evolving paradigm for stem cell biology. Cell. 2008;132(4):631-644.

2. Novershtern N, Subramanian A, Lawton LN, et al. Densely interconnected transcriptional circuits control cell states in human hematopoiesis. Cell. 2011;144(2):296-309.

3. Tenen DG, Hromas R, Licht JD, Zhang DE. Transcription factors, normal myeloid development, and leukemia. Blood. 1997;90(2):489-519.

4. Iwasaki H, Somoza C, Shigematsu H, et al. Distinctive and indispensable roles of PU.1 in maintenance of hematopoietic stem cells and their differentiation. Blood. 2005; 106(5):1590-1600.

5. Hock H, Hamblen MJ, Rooke HM, et al. Intrinsic requirement for zinc finger transcription factor Gfi-1 in neutrophil differentiation. Immunity. 2003:18(1):109-120.

6. Holtschke T, Lohler J, Kanno Y, et al. Immunodeficiency and chronic myelogenous leukemia-like syndrome in mice with a targeted mutation of the ICSBP gene. Cell. 1996;87(2):307-317.

7. Growney JD, Shigematsu H, Li Z, et al. Loss of Runx1 perturbs adult hematopoiesis and is associated with a myeloproliferative phenotype. Blood. 2005;106(2):494-504

8. Shivdasani RA, Mayer EL, Orkin SH Absence of blood formation in mice lacking the T-cell leukaemia oncoprotein tal-1/SCL. Nature. 1995;373(6513):432-434.

9. Yamanaka R, Barlow C, Lekstrom-Himes J, et al. Impaired granulopoiesis, myelodysplasia, and early lethality in CCAAT/enhancer binding protein epsilondeficient mice. Proc Natl Acad Sci USA 1997;94(24):13187-13192.

10. Zhang DE, Zhang $P$, Wang ND, Hetherington CJ, Darlington GJ, Tenen DG. Absence of granulocyte colony-stimulating factor signaling and neutrophil development in CCAAT enhancer binding protein alpha-deficient mice. Proc Natl Acad Sci USA. 1997;94(2):569-574.
11. Akagi T, Thoennissen NH, George A, et al. In vivo deficiency of both C/EBPbeta and C/EBPepsilon results in highly defective myeloid differentiation and lack of cytokine response. PLoS One. 2010; 5(11):e15419.

12. Chumakov AM, Grillier I, Chumakova E, Chih D, Slater J, Koeffler HP. Cloning of the novel human myeloid-cell-specific C/EBPepsilon transcription factor. Mol Cell Biol. 1997;17(3):1375-1386

13. Lekstrom-Himes J, Xanthopoulos KG. Biological role of the CCAAT/enhancerbinding protein family of transcription factors. J Biol Chem. 1998;273(44):28545 28548.

14. Morosetti R, Park DJ, Chumakov AM, et al A novel, myeloid transcription factor, $\mathrm{C} / \mathrm{EBP}$ epsilon, is upregulated during granulocytic, but not monocytic, differentiation. Blood. 1997;90(7):2591-2600.

15. Gombart AF, Shiohara M, Kwok SH, Agematsu K, Komiyama A, Koeffler HP Neutrophil-specific granule deficiency: homozygous recessive inheritance of a frameshift mutation in the gene encoding transcription factor CCAAT/enhancer binding protein--epsilon. Blood. 2001; 97(9):2561-2567.

16. Wada T, Akagi T, Muraoka M, et al. A novel in-frame deletion in the leucine zipper domain of C/EBPepsilon leads to neutrophil-specific granule deficiency. Immunol. 2015;195(1):80-86.

17. Gombart AF, Krug U, O'Kelly J, An E, Vegesna V, Koeffler HP. Aberrant expression of neutrophil and macrophage-related genes in a murine model for human neutrophil-specific granule deficiency. J Leukoc Biol. 2005;78(5):1153-1165

18. Grabiner BC, Blonska M, Lin PC, et al CARMA3 deficiency abrogates $G$ proteincoupled receptor-induced NF-\{kappa\}B activation. Genes Dev. 2007;21(8):984-996

19. Blonska $M$, Lin X. NF-kappaB signaling pathways regulated by CARMA family of scaffold proteins. Cell Res. 2011;21(1):5570.

20. Medoff BD, Landry AL, Wittbold KA, et al.
CARMA3 mediates lysophosphatidic acidstimulated cytokine secretion by bronchial epithelial cells. Am J Respir Cell Mol Biol. 2009;40(3):286-294.

21. Suh HC, Benoukraf T, Shyamsunder P, et al. LPS independent activation of the proinflammatory receptor Trem 1 by C/EBPepsilon in granulocytes. Sci Rep. 2017:7:46440.

22. Lara-Astiaso D, Weiner A, Lorenzo-Vivas $\mathrm{E}$, et al. Immunogenetics. Chromatin state dynamics during blood formation. Science. 2014;345(6199):943-949

23. Tanaka M, Gombart AF, Koeffler HP, Shiohara M. Expression of bactericidal/permeability-increasing protein requires C/EBP epsilon. Int I Hematol. 2007;85(4):304-311.

24. Gombart AF, Kwok SH, Anderson KL, Yamaguchi Y, Torbett BE, Koeffler HP. Regulation of neutrophil and eosinophil secondary granule gene expression by transcription factors C/EBP epsilon and PU.1. Blood. 2003;101(8):3265-3273

25. Chumakov AM, Kubota T, Walter S, Koeffler HP. Identification of murine and human XCP1 genes as C/EBP-epsilondependent members of FIZZ/Resistin gene family. Oncogene. 2004;23(19):3414-3425.

26. Khanna-Gupta A, Zibello T, Sun H, Gaines $\mathrm{P}$, Berliner N. Chromatin immunoprecipitation (ChIP) studies indicate a role for CCAAT enhancer binding proteins alpha and epsilon (C/EBP alpha and C/EBP epsilon ) and CDP/cut in myeloid maturation-induced lactoferrin gene expression. Blood. 2003;101(9):3460-3468.

27. McDonald PP, Bald A, Cassatella MA Activation of the NF-kappaB pathway by inflammatory stimuli in human neutrophils. Blood. 1997;89(9):3421-3433.

28. Cassatella MA. The production of cytokines by polymorphonuclear neutrophils. Immunol Today. 1995;16(1):21-26.

29. Kyme P, Thoennissen NH, Tseng CW, et al. C/EBPepsilon mediates nicotinamideenhanced clearance of Staphylococcus aureus in mice. J Clin Invest. 2012; 122(9):3316-3329. 